\title{
Pricing of Real-Time Applications in WiMAX Systems
}

\author{
Aymen Belghith*, Loutfi Nuaymi* and Patrick Maille* \\ *TELECOM Bretagne, France \\ 2 rue de la châtaigneraie, CS 17607, 35576 \\ Email: \{first.last $\} @$ telecom-bretagne.eu
}

\begin{abstract}
WiMAX/IEEE 802.16 Broadband Wireless Access (BWA) is a very open technology with different possible services that is likely to be widely used in Metropolitan Access Networks in the near future. While the technical aspects of that technology are now quite well established, the economic implications of the introduction of WiMAX technology have received little attention. In this paper, we consider the problem of pricing WiMAX sessions: we propose some simple pricing mechanisms aimed at coping with congestion while maintaining a sufficient quality of service and/or yielding a sufficient revenue to the operator. Our mechanisms are evaluated under a specific model of user preferences and WiMAX situations, and the implications of some parameter choices are observed.
\end{abstract}

\section{INTRODUCTION}

WiMAX technology, based on IEEE 802.16 standard [1], [2], has a very rich set of features. Indeed, it is a very promising Broadband Wireless Access (BWA) Technology, whose objective is to have a highly-efficient use of the radio resource while transmitting different types of service (with different constraints).

The IEEE 802.16 standard specifies five physical (PHY) layers: WirelessMAN-SC, WirelessMAN-SCa, WirelessMANOFDM, WirelessMAN-OFDMA, and WirelessHUMAN PHYs. We consider only the WirelessMANOFDM PHY which is based on the Orthogonal Frequency Division Multiplexing (OFDM) technology. Yet, our study could be extended to OFDMA access where OFDM symbols allocation is replaced by OFDMA subchannels allocation.

The IEEE 802.16 Medium Access Control (MAC) layer supports two modes of transmission: the point-to-multipoint (PMP) and Mesh modes. It also supports two duplex modes: the Frequency Division Duplex (FDD) and Time Division Duplex (TDD) modes. We consider the PMP transmission mode and the FDD duplex mode. Once again, our study could be extended to the TDD mode, with additional latitude.

The IEEE 802.16 MAC also specifies five types of scheduling service classes or QoS classes: Unsolicited Grant Service (UGS), real-time Polling Service (rtPS), extended real-time Polling Service (ertPS), non real-time Polling Service (nrtPS), and Best Effort (BE) QoS classes. Each QoS class has its QoS parameters and requirements. The MAC Convergence Sublayer (CS) layer makes the classification of the different users applications in those five classes.

Pricing of WiMAX is a new and interesting area. Pricing models are defined for the integration of WiMAX and Wireless
Fidelity (WiFi). For example, a strategy game used in economics, called Stackelberg leadership model, is used in [3], in order to propose a pricing model for sharing bandwidth between WiMAX and WiFi users. Other pricing models are defined only for WiMAX context. For example, two market models are defined in [4]. The Base Station (BS) allocates resources only for the residential users in the first market model. In the second model, the BS also provides services to the Small and Medium Enterprises (SME) customers. In [5], a pricing model for wireless networks users is proposed. The utility function of the proposed pricing model depends on the transmission rate and the quality of the channel. In [6], a cost-based Call Admission Control (CAC) is proposed for IEEE 802.16 WiMAX. It is based on the required bandwidth and the residual bandwidth. The competitive On-Line (COL) is used in order to have an exponential cost function of the residential bandwidth.

However, there is not yet any published pricing proposal for the different WiMAX QoS classes in the research literature. Evidently, it can be expected that WiMAX operators use different pricing strategies for the QoS classes.

In this paper, we propose pricing schemes for WiMAX. Our pricing model is designed for real-time applications and takes into account the specification of the standard. There are a large number of parameters and other models details that have to be selected for the global WiMAX pricing model. We make some decisions for these parameters in order to obtain a simplified model and some interesting first results.

This paper is organized as follows. In Section II, we present the system model. In Section III, we describe our pricing proposals for UGS and rtPS QoS classes. In Section IV, we present some simulation results. Conclusions and directions for future work are provided in Section V.

\section{System Model}

We consider a real-time environment with only the UGS and rtPS QoS classes:

- UGS: supports real-time service flows that have fixed-size data packets on a periodic basis (for circuit-type voice services for instance).

- rtPS: supports real-time service flows that generate variable data packets size on a periodic basis (for video services for instance). A minimum transmission rate 
TABLE I

NUMBER OF USEFUL BITS PER OFDM SYMBOL

\begin{tabular}{|c|c|c|c|}
\hline Modulation & Coding & Receiver SNR $(\mathrm{dB})$ & $\begin{array}{l}\text { Number of useful bits } \\
\text { per OFDM symbol }\end{array}$ \\
\hline \hline BPSK & $1 / 2$ & 3.0 & $192 * 1 * 1 / 2=96$ \\
\hline QPSK & $1 / 2$ & 6.0 & $192 * 2 * 1 / 2=192$ \\
\cline { 2 - 4 } & $3 / 4$ & 8.5 & $192 * 2 * 2 / 3=288$ \\
\hline \multirow{2}{*}{ 6-QAM } & $1 / 2$ & 11.5 & $192 * 4 * 1 / 2=384$ \\
\cline { 2 - 4 } & $3 / 4$ & 15.0 & $192 * 4 * 2 / 3=576$ \\
\hline \multirow{2}{*}{ 64-QAM } & $2 / 3$ & 19.0 & $192 * 6 * 2 / 3=768$ \\
\cline { 2 - 4 } & $3 / 4$ & 21.0 & $192 * 6 * 3 / 4=864$ \\
\hline
\end{tabular}

$\left(R_{\min }\right)$ is guaranteed, and the rate can be increased up to a maximum transmission rate $\left(R_{\max }\right)$.

In IEEE 802.16 standard and for a given system, the frame has a fixed number of OFDM symbols. The number of symbols depends on the frequency bandwidth $(B W)$, the Cyclic Prefix $(C P)$, the sampling factor $(n)$, and the frame duration.

An appropriately-designed pricing mechanism should then properly allocate those symbols among users, so as to satisfy some QoS agreements, or maximize user satisfaction or provider revenue.

However, the number of useful data bits is variable and depends on the Modulation and Coding Scheme (MCS) used by the different subscriber stations (SSs). The MCS to be used is defined by the link adaptation procedure. The choice of the appropriate MCS depends on the value of receiver Signal-toNoise Ratio (SNR). The transmitting station (the BS or the SS) switches to a more energy-efficient MCS if the SNR is good. It switches to a more robust MCS if the SNR is poor. Once the MCS is defined, the number of bits per OFDM symbol, and then the useful number of bits per frame, can be computed as shown in Table I. Values of some receiver SNR assumptions (see column 3, Table I) are proposed in Table 266 of the IEEE 802.16e amendment of the standard. The BPSK $1 / 2$ MCS is mainly used for management purposes and will not be considered in this work.

\section{PRICING MODEL}

We propose an utility function for each type of service. The utility is the difference between the price that the user is willing to pay for the service (that we call valuation or willingness to pay) and the price actually paid. The utility, valuation, and price actually paid are per unit of time.

$$
U(r)=V(r)-P(r)
$$

where:

- U: represents the utility function.

- V: represents the valuation function.

- P: represents the price actually paid function.

- $r$ : represents the throughput of the connection (expressed in bit/s).

\section{A. UGS users}

The transmission rate of the UGS connections is fixed at the beginning of the session. We consider that each UGS user $i$ is willing to pay a given price $v_{i}$ per transmitted bit per second.

$$
V_{U G S}(r)=v_{i} \cdot r
$$

We introduce heterogeneity among the UGS users by assuming that $v_{i}$ is randomly chosen for each user, according to continuous uniform distribution on the interval $\left[V 1_{U G S}\right.$, $\left.V 2_{U G S}\right]$ (in monetary units (mu) by kbit/s transmitted).

We suggest to use a pricing scheme based on two prices. When the network is not congested (i.e. when the proportion of OFDM symbols used by the UGS and rtPS calls is below a given threshold, called $t h_{U G S}$ ), the UGS calls are charged a price $P_{U G S_{-} L o w}$ per unit of transmission rate. On the other hand, during the congested periods, this price actually paid is changed to $P_{U G S_{-} H i g h}$. We consider that $P_{U G S_{-} H i g h}=\alpha_{U G S}$ * P PGS_Low.

When an UGS call starts, its price per unit of time does not change; if the call started when the network was not congested, the user continue to pay the low price during its whole call even if afterwards it is served at congestion periods.

$$
P_{U G S}(r)= \begin{cases}P_{U G S_{-} L o w} \cdot r, & \text { if } \frac{n b_{r e s}}{n b_{t o t}} \leq t h_{U G S} \\ P_{U G S_{-} H i g h} \cdot r, & \text { otherwise }\end{cases}
$$

where:

- $n b_{\text {res }}$ : represents the number of reserved symbols.

- $n b_{t o t}:$ represents the total number of symbols.

\section{B. rtPS users}

rtPS services offer a flexibility in the transmission rate. We therefore consider that users have elastic demand, i.e. their valuation function is increasing and concaves in the transmission rate $r$. We propose the following valuation function:

$$
V_{r t P S}(r)=\left\{\begin{array}{l}
v_{i} \cdot\left(\frac{-r^{2}}{R_{i \_\max }}+2 r\right), \quad \text { if } r \leq R_{i_{-} \max } \\
v_{i} \cdot R_{i \_ \text {max }}, \quad \text { otherwise }
\end{array}\right.
$$

We also introduce heterogeneity among the rtPS users by assuming that $v_{i}$ is randomly chosen for each user, according to continuous uniform distribution on the interval $\left[V 1_{r t P S}\right.$, $V 2_{r t P S}$ ] (in mu by kbit/s transmitted).

A minimum transmission $R_{\text {min }}$ rate is guaranteed to rtPS users upon connection. We suggest that this minimum rate is provided (when there are enough OFDM symbols remaining to serve this call) at a fixed unit price that we denote by $P_{r t P S_{-} m i n}$, independently of the congestion. On the other hand, the rtPS QoS Class offers the possibility to extend the transmission rate up to $R_{\max }$. We propose that the additional transmission rate is sold $P_{r t P S_{-} a d d}$ that could increase with congestion. This way, during congestion periods, rtPS users will be elicited to reduce their transmission rate close to $R_{\text {min }}$ due to high prices. Formally, a user $i$ with transmission rate $r$ that belongs to $\left[R_{\min }, R_{\max }\right]$ is therefore charged a price:

$$
P_{r t P S}(r)=P_{r t P S \_m i n} \cdot R_{m i n}+P_{r t P S \_a d d} \cdot\left(r-R_{\text {min }}\right)
$$


The price for extra rate is equal to:

$$
P_{r t P S \_a d d}=\frac{C_{r t P S}}{n b_{r e m \_m i n}}
$$

Where:

- $C_{r t P S}$ : represents a pricing constant fixed by the operator.

- $n b_{\text {rem_min }}$ : represents the number of OFDM symbols remaining after all the UGS users are served and the rtPS users get their minimum transmission rate.

\section{Simulation Results}

\section{A. Simulation model}

This section describes the situation we have considered to simulate the behavior of a WiMAX system under the pricing schemes described in the previous section. In this work, we consider the OFDM PHY layer used in the PMP topology and FDD mode. The number of OFDM symbols per frame $\left(N_{O F D M}\right)$ is constant and depends on the frame duration and the OFDM symbol duration. According to [7], the OFDM symbol duration is computed as follows:

$$
\text { OFDM symbol Duration }=\frac{1+G}{n \cdot \frac{B W}{N_{F T T}}}
$$

where:

- $G$ : represents the ratio of the guard time to the useful symbol time.

- $n$ : represents the the sampling factor.

- $B W$ : represents the channel bandwidth.

- $N_{F T T}$ : represents the total number of subcarriers. For the OFDM PHY, the total number of subcarriers is equal to $256\left(N_{F T T}=256\right)$.

For the following parameter values: $B W=7 \mathrm{MHz}, n=8 / 7$, $G=1 / 8$, and frame duration $=10 \mathrm{~ms}$ obtain an OFDM symbol duration equal to $35.86 \mu$ s and $N_{O F D M}$ equal to 278 symbols. The simulation duration is equal to $300 \mathrm{~s}$.

In our simulation framework, we consider Poisson arrivals of each class of customer UGS and rtPS, with respective arrival rate $\lambda_{U G S}=\lambda_{r t P S}=1 / 30 s^{-1}$. We assume that the durations of the UGS and rtPS sessions follow exponential distributions with respective mean $\mu_{U G S}=\mu_{r t P S}=5$ minutes.

We consider three types of UGS users: $10 \mathrm{kbit} / \mathrm{s}, 50 \mathrm{kbit} / \mathrm{s}$, and $200 \mathrm{kbit} / \mathrm{s}$, that represent $25 \%, 25 \%$, and $50 \%$ of the UGS users, respectively. We also consider four types of rtPS users, with respective minimal data rate values (in kbit/s) $R_{\min }=30$, 50, 100, 200 and maximal data rate values (in kbit/s) $R_{\max }$ $=100,300,500,800$. We assume that each of these $\left[R_{\min }\right.$, $R_{\text {max }}$ ] couples is chosen by $25 \%$ of the rtPS users.

The user SNR values are randomly chosen at the beginning of the session, considering a simple cellular model with three cells per cluster: the SNR distribution can then be obtained using classical results of the SNR repartition in cellular networks [8]. Having the SNR values, the MCS used is directly deduced from Table I. The distribution of SNRs (and then MCSs) is given in Table II.

The valuation function (in mu by $\mathrm{kbit} / \mathrm{s}$ transmitted) of the UGS and rtPS users are according to continuous uniform
TABLE II

DISTRIBUTION OF THE MCSS FOR ARRIVING USERS

\begin{tabular}{|c|c|}
\hline SNR range $(\mathrm{dB})$ & Proportion of users \\
\hline \hline$[6,8.5)$ & $30 \%$ \\
\hline$[8.5,11.5)$ & $10 \%$ \\
\hline$[11.5,15)$ & $20 \%$ \\
\hline$[15,19)$ & $15 \%$ \\
\hline$[19,21)$ & $5 \%$ \\
\hline$[21,+\infty)$ & $20 \%$ \\
\hline
\end{tabular}

distributions on the intervals $\left[V 1_{U G S}, V 2_{U G S}\right]$ and $\left[V 1_{r t P S}\right.$, $\left.V 2_{r t P S}\right]$, respectively. In the rest of the paper, we assume that $V 1_{U G S}=V 1_{r t P S}=0.5$ and $V 2_{U G S}=V 2_{r t P S}=1$.

Since the UGS and rtPS QoS classes are designed for realtime applications, an admission control mechanism is required. An UGS (resp. rtPS) customer is admitted only if there are enough OFDM symbols, taking into account its MCS used, for its data rate (resp. for its minimum data rate $R_{m i n}$ ).

\section{B. Investigation of $P_{\text {ref }}$}

In this paper, we assume that $P_{U G S_{-} \text {Low }}=P_{r t P S_{-} \text {min }}=$ $P_{\text {ref }}$. For the UGS pricing model, we adopt $\alpha_{U G S}=2$ $\left(P_{U G S_{-} H i g h}=2 * P_{\text {ref }}\right), t h_{U G S}=0.5$. For the rtPS pricing model, we adopt $C_{r t P S}=10$.

Fig. 1 shows the mean throughput of the UGS and rtPS connections as a function of $P_{\text {ref }}$. The throughput of the UGS connections is constant when $P_{\text {ref }}$ increases and $P_{\text {ref }}<0.25$. This is because the highest price actually paid $\left(P_{H i g h}=2 *\right.$ $\left.P_{\text {ref }}=0.5\right)$ is lower or equal than the lowest call valuation (belongs to $\left[V 1_{U G S}, V 2_{U G S}\right]$ ).

We notice that the throughput of the UGS connections decreases when $P_{\text {ref }}$ increases and $P_{\text {ref }}>0.25$. This is due to the high price actually paid and so some UGS users cannot pay to reserve for their data rates. When $P_{\text {ref }}$ is upper than $V 2_{U G S}\left(V 2_{U G S}=1\right)$, the throughput is equal to 0 because the utility function is always negative.

The throughput of the rtPS connections also constant when $P_{\text {ref }}$ increases and $P_{\text {ref }}<0.9$. It decreases when $P_{\text {ref }}$ increases and $P_{\text {ref }}>0.9$. Indeed, the rtPS users cannot extend their transmissions rate to $R_{\max }$ and are be served,when the utility function $>0$, at a rate $r$ in $\left[R_{\min }, R_{\max }\right]$ (depending on the price actually paid).

Fig. 2 shows the mean blocking rate of the UGS and rtPS connections as a function of $P_{\text {ref }}$. A connection is blocked when there are not enough symbols (noted "symbols $<0$ ") or the price actually paid is upper than the price that the user is willing to pay (noted "utility < 0"). The blocking rate (because "utility $<0$ ") of the UGS connections increases when $P_{\text {ref }}$ increases and this is due to the high price actually paid. When $P_{\text {ref }}$ is upper than $V 2_{U G S}$, the blocking rate (because "utility < $<$ ") becomes constant because all the UGS connections are blocked (UGS throughput $=0$, see Fig. 1).

When $P_{\text {ref }}>0.75$, rtPS connections can be blocked because of the high price actually paid. This happens when rtPS connections cannot pay to reserve $R_{\min }$. 


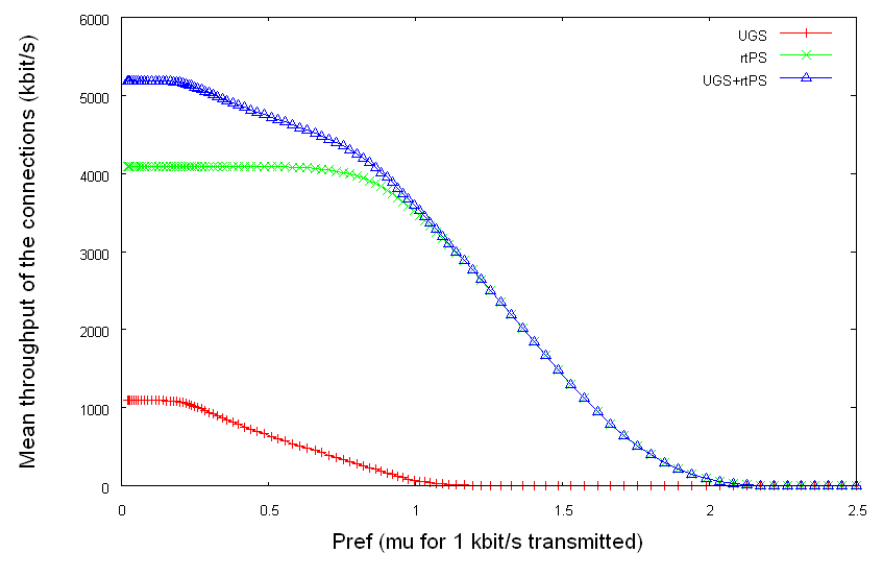

Fig. 1. Mean throughput versus $P_{\text {ref }}$

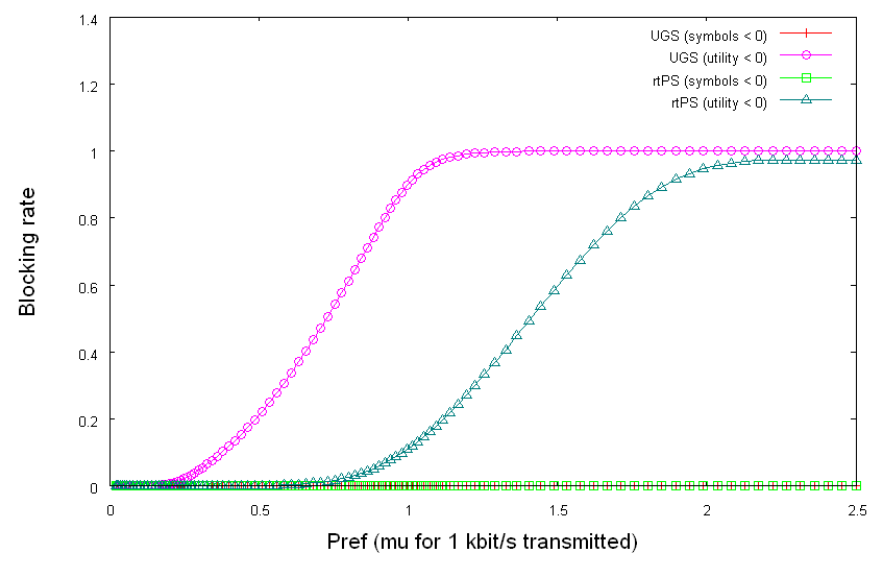

Fig. 2. Mean blocking rate versus $P_{\text {ref }}$

We observe that the blocking rate (because "symbols $<0$ ") of UGS and rtPS connections are almost equal to 0 . Indeed, the throughput decreases when the blocking rate is $>0$. Since, when $P_{\text {ref }}$ is low, the throughput of the UGS and rtPS connections do not decrease (Fig. 1), the blocking rate (because "utility $<0$ " and "symbol $<0$ ") of the UGS and rtPS connections have to be equal to 0 .

Fig. 3 shows the mean number of the UGS and rtPS connections in the system as a function of $P_{\text {ref }}$. The mean number of connections in the system decreases when $P_{\text {ref }}$ increases. This is due to the increase of the blocking rate. We observe that $P_{\text {ref }}$ has less influence on the rtPS connections. Indeed, an UGS connection is blocked when it cannot pay to reserve its data rate. On the other hand, an rtPS connection is served even if it cannot pay to reserve its $R_{\max }$. It is served when it can pay to reserve $r$ in $\left[R_{\min }, R_{\max }\right]$.

Fig. 4 shows the mean revenue from the UGS and rtPS connections as a function of $P_{\text {ref }}$. The revenue from the UGS connections increases when $P_{\text {ref }}$ increases without exceeding 0.25 . This is because no UGS connections is blocked (blocking rate $=0$ when $P_{\text {ref }}<0.25$, see Fig. 2) and the price actually paid increases when $P_{\text {ref }}$ increases. When $P_{\text {ref }}$ increases and $P_{\text {ref }}>0.25$, the revenue from the UGS connections

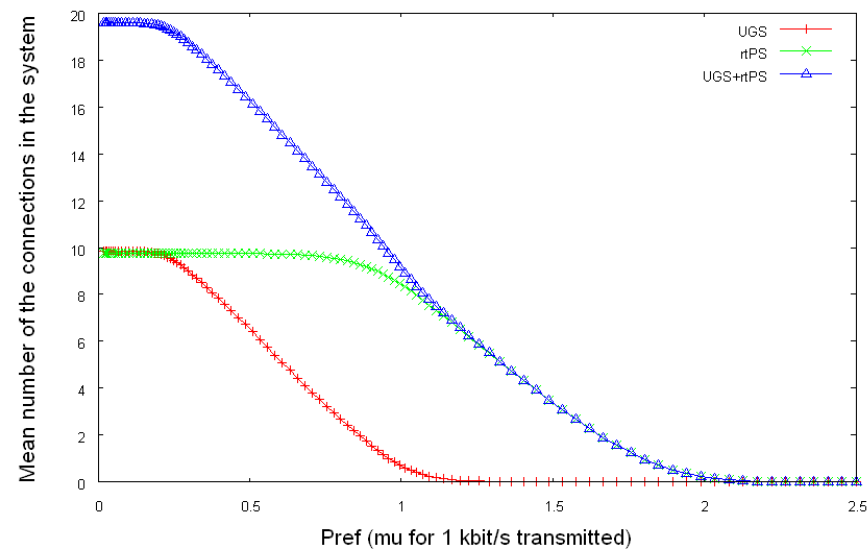

Fig. 3. Mean number of the connections in the system versus $P_{\text {ref }}$

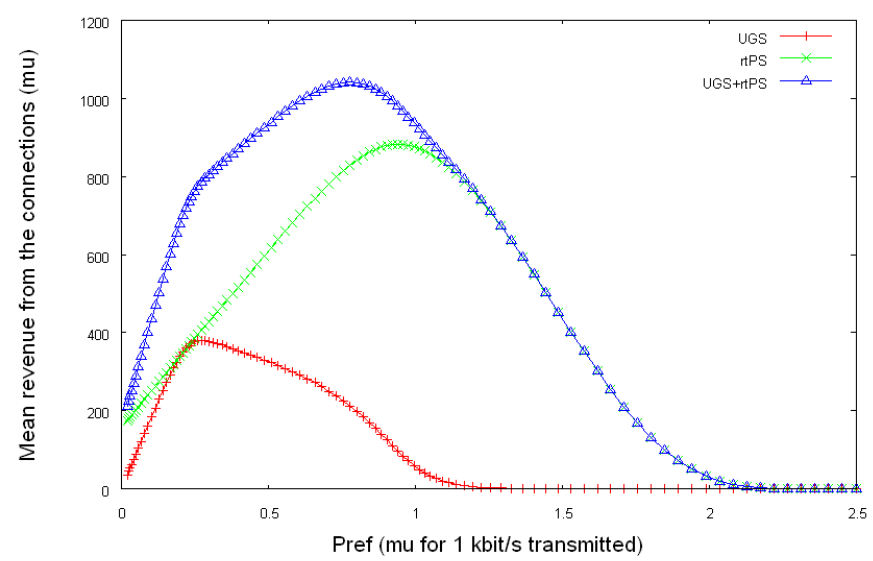

Fig. 4. Mean Revenue from the connections versus $P_{r e f}$

decreases. This is due to the high increase of the blocking rate.

When $P_{\text {ref }}$ increases, the revenue from the rtPS connections increases when $P_{\text {ref }}<1$ and decreases when $P_{\text {ref }}>1$. The behavior of the revenue depends on the increase of the price actually paid and the increase of the blocking rate.

We note that the total revenue increases when $P_{\text {ref }}<0.9$, otherwise, it decreases. If the objective of a pricing scheme is only to maximize the network revenue, we choose 0.9 as a value of $P_{\text {ref }}$. In addition of the maximization of the revenue, we can add some constraints to our pricing model. For example, if we add the minimization of the blocking rate and the maximization of the total throughput, we assign 0.25 to $P_{\text {ref }}$.

\section{Investigation of $C_{r t P S}$}

We recall that the price actually paid for rtPS connections (specially the price for extra rate) depends on $C_{r t P S}$ (see (6) and (5)). In this Section, we adopt $P_{U G S_{-} L o w}=P_{r t P S_{-} \text {min }}=$ $P_{\text {ref }}=0.25, \alpha_{U G S}=2$, and $t h_{U G S}=0.5$.

Fig. 5 shows the mean throughput of the UGS and rtPS connections as a function of $C_{r t P S}$. The throughput of the rtPS connections decreases when $C_{r t P S}$ increases. This is due to 


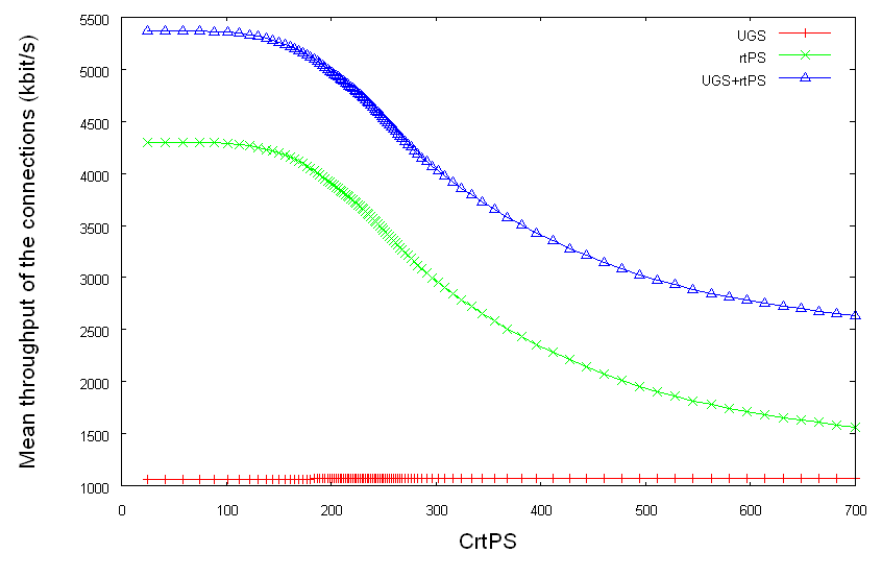

Fig. 5. Mean throughput versus $C_{r t P S}$

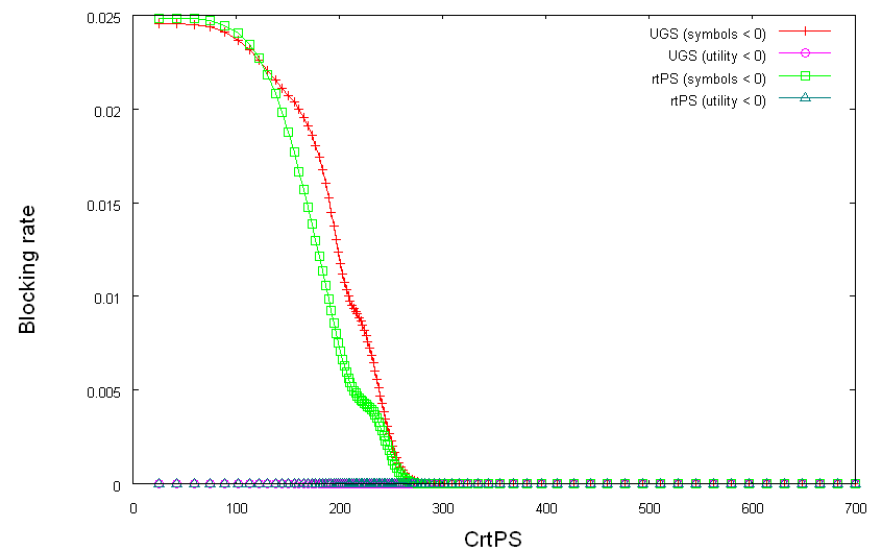

Fig. 6. Mean blocking rate versus $C_{r t P S}$

the increase of the price actually paid for the extra rate $\left(r_{\text {extra }}\right)$ that belongs to $\left[0, R_{\max }-R_{\min }\right]$.

The throughput of the UGS connections slightly increases when $C_{r t P S}$ increases. This is because the remaining symbols increases when the symbols used by the rtPS connections decreases.

Fig. 6 shows the mean blockage rate of the UGS and rtPS connections as a function of $C_{r t P S}$. The blocking rate (because "utility < 0") of the UGS connections is equal to 0 because the minimum price that an UGS user is willing to pay $\left(V 1_{U G S}=0.5\right)$ is upper or equal than the maximum price that can actually paid $\left(P_{U G S_{-} H i g h}=2 * P_{\text {ref }}=0.5\right)$.

The blocking rate (because "utility $<0$ ") of the rtPS connections is equal to 0 because all the rtPS connections can pay to reserve $R_{\text {min }}\left(P_{r t P S} S_{-m i n}\right.$ is independent of $\left.C_{r t P S}\right)$.

The blocking rate (because "symbols < 0 ") of the UGS and rtPS becomes equal to 0 when $C_{r t P S}$ exceeds a certain value $\left(C_{r t P S}=250\right)$. This is because of the decrease of the total throughput when $C_{r t P S}$ increases. Then, there are more available symbols in the system.

Fig. 7 shows the mean number of the UGS and rtPS connections in the system as a function of $C_{r t P S}$.

The mean number of the UGS and rtPS connections in the

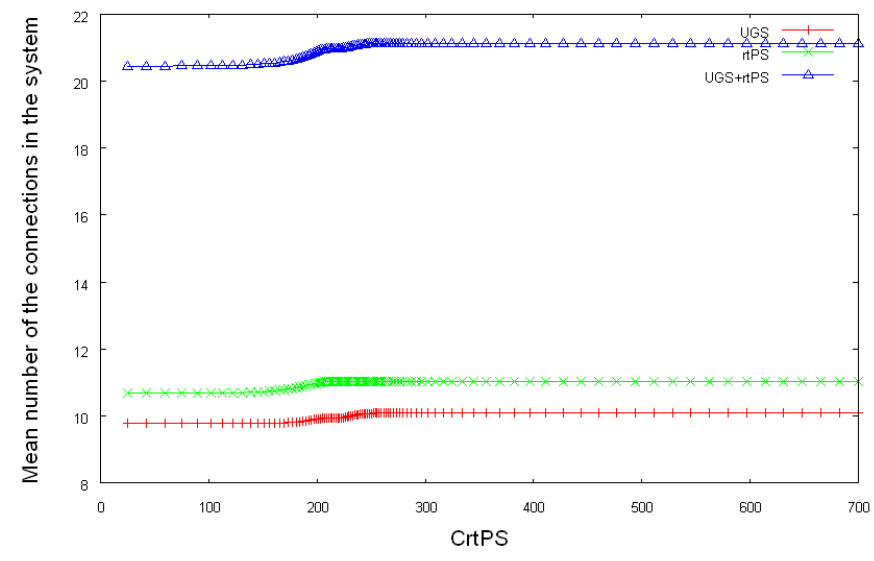

Fig. 7. Mean number of connections in the system versus $C_{r t P S}$

system increases slightly when $C_{r t P S}$ increases and $C_{r t P S}<$ 250. Otherwise, it becomes constant. Indeed, this directly depends on the blocking rate. All the connections are served when $C_{r t P S}>250$. Therefore, the total number of the served connections cannot increase even if there are more available symbols in the system.

Fig. 8 shows the mean revenue from the UGS and rtPS connections as a function of $C_{r t P S}$. We observe that the revenue from the UGS connections is almost constant because the price actually paid for this kind of user is independent of $C_{r t P S}$.

The revenue from the rtPS connections increases when $C_{r t P S}$ increases and $C_{r t P S}<250$. Indeed, although the decrease of the throughput of the rtPS connections, the price actually paid for the extra data increases and the rtPS users are still able to pay to reserve an additional rate (its depends on the valuation function).

When $C_{r t P S}>250$, the revenue from the rtPS connections decreases when $C_{r t P S}$ increases. This is because the price actually paid for extra data is too high that the rtPS users restricts on a throughput closer than their $R_{\min }$.

Therefore, we can assign 250 to $C_{r t P S}$ if our single aim is to maximize the total revenue. We also choose 250 as a value of $C_{r t P S}$ if we add the minimization of the blocking rate and secondly the maximization of the throughput as constraints.

\section{CONCLUSION}

In this paper, we have proposed a pricing scheme for WiMAX systems. This scheme is designed for the UGS and rtPS QoS classes that represent the real-time applications in WiMAX. Moreover, our proposed mechanism is easily understandable by users and reasonably fair, and could therefore be implemented in some WiMAX systems. We have set a precise simulation framework to study the implications of our proposals, and exhibited some good properties of our pricing model in terms of revenue maximization, throughput and user satisfaction (via the blocking rate). These proposals include many parameters, which gives way to interesting optimizations. The choice of the system parameters depends on 


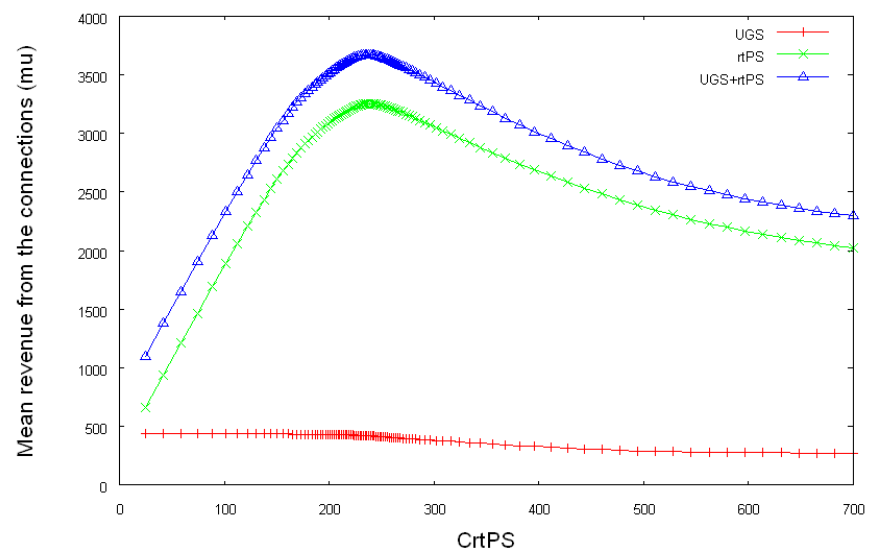

Fig. 8. Mean revenue from the connections versus $C_{r t P S}$

the optimization criteria. As other directions for future work, we would like to turn our attention to extend our pricing model in order to support non-real-time applications in WiMAX systems and study its behavior in such context.

\section{ACKNOWLEDGMENT}

This work is supported by the Britanny (Bretagne) Region in the framework of Op WiMAX Project.

\section{REFERENCES}

[1] IEEE 802.16-2004, IEEE Standard for local and metropolitan area networks, Air Interface for Fixed Broadband Wireless Access Systems, Oct 2004.

[2] IEEE 802.16e, IEEE Standard for local and metropolitan area networks, Air Interface for Fixed Broadband Wireless Access Systems, Amendment 2: Physical and Medium Access Control Layers for Combined Fixed and Mobile Operation in Licensed Bands and Corrigendum 1, Feb 2006 (Approved: 7 Dec 2005).

[3] D. Niyato and E. Hossain, "Integration of WiMAX and WiFi: Optimal Pricing for Bandwidth Sharing", IEEE Communications Magazine, vol. 45, no. 5, pp. 140 - 146, 5 May 2007.

[4] "Business Case Models for Fixed Broadband Wireless Access based on WiMAX Technology and the 802.16 Standard", White Paper, WiMAX Forum, 10 October 2004.

[5] J.-W. Lee, M. Chiang, and A. R. Calderbank, "Price-based distributed algorithms for rate-reliability tradeoff in network utility maximization", IEEE Journal on Selected Areas in Communications, vol. 24, no. 5, pp. 962 - 976, May 2006.

[6] B.-J. Chang Y.-L. Chen, and C.-M. Chou, "Adaptive Hierarchical Polling and Cost-based Call Admission Control in IEEE 802.16 WiMAX", Wireless Communications and Networking Conference, 2007, WCNC 2007, pp. 1954 - 1958, Hong Kong, 11-15 March 2007.

[7] L. Nuaymi, WiMAX: Technology for Broadband Wireless Access, Wiley, January 2007.

[8] X. Lagrange, "CIR cumulative distribution in a regular cellular network", ENST, Tech. Rep., 2000. 